\title{
Acute Toxicity and Local Response using Three Fractions of High Dose Rate (HDR) Brachytherapy for Curative Treatment of Carcinoma Cervix
}

\author{
Nasir Ali, Bilal Mazhar Qureshi, Ahmed Nadeem Abbasi, Asim Hafiz, Benazir Mir Khan and Abdul Qadir Jangda \\ Radiation Oncology Section, Department of Oncology, The Aga Khan University, Karachi, Pakistan
}

\begin{abstract}
To determine the acute vaginal mucosal toxicity and clinical response of cervical cancer after definitive treatment with external beam radiotherapy (EBRT) and three fractions each of 8 Fray (Gy) high dose rate intracavitary brachytherapy (HDR-BT).

Study Design: Descriptive study.

Place and Duration: Radiation Oncology Section, Department of Oncology, The Aga Khan University Hospital, Karachi, Pakistan from January 2008 till December 2015.

Methodology: Protocol was formulated for carcinoma cervix to complete treatment in 7 weeks. Patients were treated with chemotherapy and pelvic EBRT to a total dose of 45 Gy/25 fractions, followed by three intracavitary HDR brachytherapy fractions of 8 Gy each. Vaginal toxicity and local clinical response was assessed at the end of treatment, at 4 and 8 weeks.

Results: A total of 57 patients were treated with HDR brachytherapy and 49 patients were evaluated for assessment of toxicity and response. According to FIGO staging system, two had stage IB2, one had IIA, thirty-six had IIB, seven had IIIB, one had IVA disease and two had IVB with para aortic nodes. Concurrent gemcitabine and cisplatin were given to $26(46 \%)$; whereas, $28(49 \%)$ received concurrent cisplatin alone. Grade III acute vaginal mucosal toxicity was seen in 52 and Grade IV acute vaginal mucosal toxicity was observed in 08 patients. At completion of treatment, 40 patients had complete clinical response, at 4 weeks follow-up, complete regression of disease was found in 3 more and at 8 weeks none had clinical residual disease.

Conclusion: This regimen of HDR brachytherapy treatments is feasible, efficacious, and well-tolerated for carcinoma cervix in a setup with cost constraints. Long term toxicity and disease control remains to be reported with longer follow-up.
\end{abstract}

Key Words: Carcinoma cervix, High dose rate brachytherapy, Acute toxicity, Local response, External beam radiation therapy, Intracavitary brachytherapy.

How to cite this article: Ali N, Qureshi BM, Abbasi AN, Hafiz A, Khan BM, Jangda AQ. Acute Toxicity and Local Response using Three Fractions of High Dose Rate (HDR) Brachytherapy for Curative Treatment of Carcinoma Cervix. J Coll Physicians Surg Pak 2020; 30(09):974-979.

\section{INTRODUCTION}

Cervical cancer is a disease of developing countries lacking human papilloma virus screening; and ranks third most common malignancy among females in Pakistan. ${ }^{1,2}$ The standard treatment for locally advanced cervical cancer is cisplatin based concurrent chemoradiotherapy and brachytherapy. ${ }^{3}$ While brachytherapy is an integral part in the management of cervical cancer, it is also established that entire treatment must be completed in a specified time of 7-8 weeks. ${ }^{4}$

Correspondence to: Dr. Bilal Mazhar Qureshi, Department of Radiation Oncology, The Aga Khan University, Stadium Road, Karachi, Pakistan

E-mail: bilal.qureshi@aku.edu

Received: June 08, 2018; Revised: January 03, 2020;

Accepted: January 30, 2020

DOI: https://doi.org/10.29271/jcpsp.2020.09.974
In a series of 113 women diagnosed with stage IB to IIIB cervical carcinoma, at a median follow-up of 26 months, time to completion of treatment more than 56 days was associated with a higher rate of disease progression within the pelvis $26 \%$ versus $9 \% .{ }^{5}$ Though American Brachytherapy Society (ABS) has recommended fractionation schedules of high dose rate brachytherapy (HDR-BT) according to stage of disease, still there is wide variation in prescribed fractionation schedule of HDR-BT in literature. ${ }^{6}$ At the time of inception of HDR-BT services at the study centre in late 2007 , the authors empirically formulated a three fractions schedule of brachytherapy with the intention to complete entire treatment in 7 weeks duration, used as three fractions HDR brachytherapy of $8 \mathrm{~Gy}$ each in these patients. It was important to evaluate the grade of vaginal mucosal toxicity and response of local disease at completion of treatment and on early follow up by clinical examination for this treatment.

The aim of this study was to report acute vaginal mucosal toxicity and clinical response of cervical cancer after definitive 
treatment with external beam radiotherapy (EBRT) and three fractions each of 8 Gray (Gy) high dose rate intracavitary brachytherapy (HDR-BT).

\section{METHODOLOGY}

After the inception of HDR-BT services at the Aga Khan University, a road map was developed for the treatment of cervical cancer patients with the aim to complete the treatment in seven weeks. It was mandatory for all patients to undergo pelvic examination under general anesthesia for staging and biopsy of cervical mass and cystoscopy and procto-sigmoidoscopy whenever required; systemic staging work up comprised of contrast enhanced CT scan of chest abdomen and pelvis. Therefore, all the patients with locally advance cervical cancer were managed, after pretreatment evaluation with the treatment scheme mentioned below, from January 2008 till December 2015.

After staging and pre-treatment workup, patients started treatment with external beam radiation therapy (EBRT) and concurrent weekly chemotherapy, preferably on first day. Patients were evaluated and considered for smit sleeve insertion in fourth week of treatment under general anesthesia by gynaeoncologist. First fraction of brachytherapy was delivered during fourth or fifth week of treatment before completion of external beam radiation therapy. Patients did not receive EBRT on the day of brachytherapy.

All patients were treated with concurrent chemotherapy and EBRT and HDR-BT. CT based 3-D conformal EBRT planning was preferred. The dose of EBRT consisted of $4500 \mathrm{cG}$ over 25 fractions on 18MV linear accelerator over five weeks. Superior limit of the anterior and posterior field was kept at L4-5 junction, inferior border was kept $2-3 \mathrm{~cm}$ below the palpable tumor in vagina or at base of obturator foramen, whatever was inferior. Laterally, the field extended $2 \mathrm{~cm}$ to the bony margin of pelvis. Lateral field borders were placed at the $2^{\text {nd }}$ and $3^{\text {rd }}$ sacral space at posterior margin and anterior border was placed at the anterior cortex of pubic symphesis. Cranio-caudal length of lateral field was equal to the length of anterio-posterior fields. However, in patients with para aortic lymphadenopathy superior border of the fields was placed at top of twelfth thoracic vertebra. Multi leaf collimators were used to block the normal tissue as much as possible without compromising the dose to the planning target volume (PTV).

Concurrent chemotherapy during EBRT was part of treatment plan. Patients receiving single agent cisplatin got dose of 40 $\mathrm{mg} / \mathrm{m}^{2}$ every week. Patients receiving doublet chemotherapy had cisplatin $40 \mathrm{mg} / \mathrm{m}^{2}$ and gemcitabine $125 \mathrm{mg} / \mathrm{m}^{2}$ on weekly basis. This was followed by adjuvant two cycles of same chemotherapy, i.e. cisplatin $50 \mathrm{mg} / \mathrm{m}^{2}$ on day 01 , gemcitabine $1000 \mathrm{mg} / \mathrm{m}^{2}$ on day 01 and 08 , cycle to be repeated at 3 weeks interval. One patient had second primary of locally advanced left breast cancer as well, so she received carboplatin at the dose of area under the curve (AUC) 5, and paclitaxel $80 \mathrm{mg} / \mathrm{m}^{2}$ weekly during pelvic EBRT, and after completion of radiation therapy she received four cycles of Adriamycin and cyclophos- phamide chemotherapy then subjected to left modified radical mastectomy followed by left chest wall and regional nodal radiation therapy.

HDR brachytherapy was delivered at 8 Gy per fraction with weekly interval, first fraction of brachytherapy was delivered during last week of EBRT and, EBRT fraction was not delivered on the day of HDR-BT as shown in Table I of road map to cervical cancer treatment. Irredium-192 HDR treatment was used with GammaMed machine. The selection of applicator was done by the radiation oncologist on vaginal examination before brachytherapy procedure between either Fletcher Suit Delclos Tandem and Ovoid applicators or Tandem and Ring applicators. HDR-BT dose was prescribed at right and left point A. Insertion of applicators were done under general anesthesia. Careful vaginal packing was done to displace the bladder and rectum away from the applicators (Figures 1 and 2). HDR treatment planning was performed on Abacus treatment planning in first half of the total duration and on Eclipse planning system in second half of study period.

Acute vaginal mucosa toxicity was recorded at conclusion of HDR brachytherapy, and four and eight weeks after completion of treatment according to the Common Terminology Criteria for Adverse Events (CTCAE) Version 4.0. ${ }^{7}$ Toxicity assessment results were recorded in patient's confidential records.

Tumor bi-dimensional measurements were recorded at start of treatment and response was done by bi-dimensional measurement of cervical mass by the same physicians after completion of treatment. During concurrent chemoradiotherapy complete blood count and serum creatinine were checked in all the patients at weekly interval.

After completion of treatment, patients were followed every two months in first year, three months in second and 6 monthly in the subsequent years. Follow up was done by physical examination, cervical cytology, and when required by pelvic MRI or CT scans. All patients were instructed to use vaginal dilator to preventvaginal adhesions and stenosis.

Patient who received EBRT in an outside centre (five patients) were censored form this analysis of toxicity assessment. Statistical Analysis was done using IBM Statistics 20.

\section{RESULTS}

A total of 57 patients received brachytherapy at this Institute from January 2008 till December 2015. The mean age of patients was $53 \pm 12.5$ years ranging from $24-83$. The mean duration of overall treatment time was 56 days. This group had squamous cell carcinoma in 51 (90\%) and adenocarcinoma in 5 (9\%) of patients. Only one patient was diagnosed to have poorly differentiated small cell carcinoma. All the patients were staged according to FIGO staging system, 40 (70\%) patients had FIGO Stage IIb disease. Three patients had FIGO stage IVb disease by virtue of paraortic lymphadenopathy, without any distant metastasis. 
Table I: Road map to cervical cancer definitive treatment.

\begin{tabular}{|l|c|c|c|c|c|c|c|}
\hline Weeks & Monday & Tuesday & Wednesday & Thursday & Friday & Saturday & Sunday \\
\hline 1 & CRT & RT & RT & RT & RT & Off \\
\hline 2 & CRT & RT & RT & RT & RT & Off \\
\hline 3 & CRT & RT & RT & RT & RT & Off \\
\hline 4 & CRT & RT & RT & RT & Smit's sleeve insertion & Off & Off \\
\hline 5 & CRT & RT & RT & RT & HDR 1 & Off & Off \\
\hline 6 & CRT & RT & Off & Off & HDR 2 & Off & Off \\
\hline 7 & Off & Off & Off & Off & HDR 3 & Off & Off \\
\hline CRT: Chemoradiation therapy; $R$ Radiation therapy; HDR: High dose rate. & & &
\end{tabular}

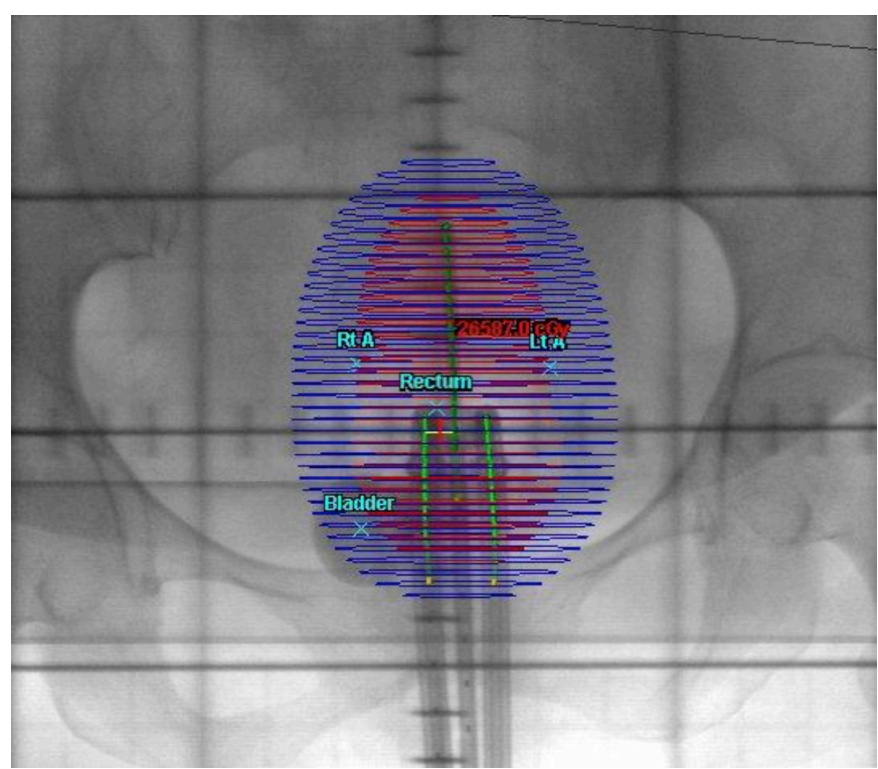

Figure 1: Anterior simulation X-Ray (inverted) image after insertion of intracavitary applicators including a tandem and two ovoids. Blue colour depicts $50 \%$ isodose coverage and red indicates $100 \%$ ( $8 \mathrm{~Gy}$ ) coverage. Central green line denotes the Irridium-192 source stepping position in tandem and the two lateral green lines denote the source stepping position in ovoids. Point $A$ on right (Rt $A$ ) and left (Lt $A)$, bladder and rectal points are marked on this simulation X-Ray image.

Out of 57 patients, $50(88 \%)$ received external beam radiation therapy in this study. Conventional two-dimensional (2D) techniques four-field box was used in five (9\%) patients. Three-dimensional conformal planning was used for 45 (79\%) of the patients using Varian eclipse treatment planning system. Seven (12\%) patients were referred to this facility only for brachytherapy and are included in this analysis. Concurrent weekly chemotherapy was given to 55 of 57 patients. Cisplatin was given to 28 (49\%) patients and combination of concurrent gemcitabine and cisplatin was given to $26(46 \%)$ patients. Two patients were not given chemotherapy because of advance age and poor performance status. Tandem and Ovoid Fletcher-Suit Delclos-style Applicator Set was used for brachytherapy in 48 (84\%) patients and Ring Applicator was used in 9 (16\%) of patients.

At the end of treatment, vaginal mucosal toxicity was recorded for all of the patients. Grade II vaginal mucositis was seen in 11 (19\%) patients. Grade III mucositis was most common, seen in 42 (73\%). Only four (7\%) patients had grade IV vaginal mucositis at the end of treatment.

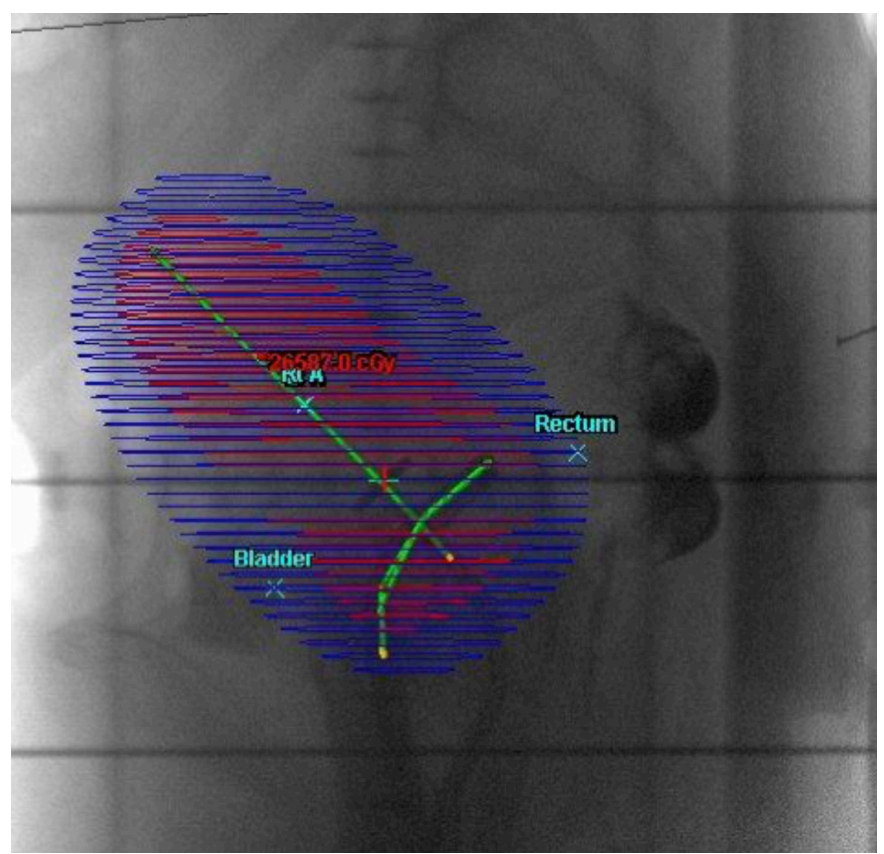

Figure 2: Lateral simulation X-Ray (inverted) image after insertion of intracavitary applicators including a tandem and two ovoids (overlapping. Blue colour depicts $\mathbf{5 0} \%$ isodose coverage and red indicates $100 \%(8 \mathrm{~Gy})$ coverage. The longer green line denotes the Irridium-192 source stepping position in tandem and the two inferior green lines denote the source stepping position in ovoids. Point $A$ on right (Rt A) and left (Lt A), bladder and rectal points are marked on this simulation X-Ray image. Contrast can be seen in rectum.

All patients were evaluated in clinic four weeks after the last brachytherapy. Eighteen (32\%) patients were observed to have grade I vaginal mucositis, grade II mucositis was present in 37 (65\%) and only two (3.5\%) patients had grade III vaginal mucositis. Eight weeks after completion of treatment none of the patients had significant vaginal toxicity.

\section{DICSUSSION}

Due to lack of vaccination, screening and awareness, females with cervical cancer present with locally advanced disease in Pakistan. ${ }^{8,9}$ In a case series from Pakistan, Hashmi et al. has reported $66 \%$ of their patients belonging to stage $\mathrm{IIb}$, which is similar to this study's experience of $70 \%{ }^{10}$ Brachytherapy is an integral component in the management of locally advanced cervical cancer as part of definitive treatment with chemo-radiation. ${ }^{3}$ Traditionally, low dose rate brachytherapy regimens have been used. However, in more and more facilities LDR is being replaced by HDR units on 
account of less cost of treatment, less potential complications, equivalent control and survival rates in favor of HDR brachytherapy regimens. ${ }^{11-15}$ Till to date, there is wide variation in the dose schedule of HDR treatment. ${ }^{16}$ Optimum dose schedule has not yet been defined, and American Brachytherapy Society authors carefully stated that proposed recommendations have not tested clinically. ${ }^{4}$

The clinicians opted three fractions of HDR brachytherapy of $8 \mathrm{~Gy}$ each. The aim was to reduce the cost of treatment and timely completion of chemoradiation therapy, but at the same time vaginal mucosa toxicity was a concern. Therefore, vaginal mucosal toxicity was graded according to CTCAE system and recorded at completion of HDR brachytherapy, at 4 weeks and at eight weeks follow up. In a prospective phase III trial single agent cisplatin was compared with doublet of cisplatin and gemcitabine and all patients received a total dose of 5040 cGy EBRT plus LDR brachytherapy. ${ }^{12}$ It showed increased systemic toxicity using doublet chemotherapy regimen. Systemic toxicity in these patients was not higher that reported in that trial because we used 4500 cGy over 25 fractions of EBRT plus HDR brachytherapy in the two arms of chemotherapy regimens. Since 2007, the dose fractionation has been three fractions of $8 \mathrm{~Gy}$ each at point A. Vaginal grade III mucosal toxicity was seen in equal proportion in both the chemotherapy regimens used (weekly concurrent cisplatin chemotherapy 40 $\mathrm{mg} / \mathrm{m}^{2}$ in 25 patients or cisplatin $40 \mathrm{mg} / \mathrm{m}^{2}$ and gemcitabine $125 \mathrm{mg} / \mathrm{m}^{2}$ in other 25 patients), only in one patient from Kenya who received concurrent cisplatin chemotherapy alone, had iatrogenic longitudinal tear in vaginal mucosa in first fraction of HDR treatment, so she was not given subsequent two fractions of HDR and given boost of 14 Gy with EBRT. Hashmi et al has reported complete response in 54\% and partial response in $38 \%$ patients with concurrent cisplatin chemotherapy with only $43 \%$ patients having vaginal mucositis. ${ }^{16}$ However, the timing of recording the acute toxicity has not been elaborated. In the current study the toxicity has been reported at three different intervals.

Rectal and bladder point doses according to the ICRU calculation points at level of external os were kept less than $60 \%$ of dose at point $A .{ }^{13}$ After two or three fractions, toxicity has been reported similar to patients in the present study. In a report by Petereit et al., authors were unable to identify a total threshold dose or the number of fractions used that could be taken as a factor contributing to the tissue complications. ${ }^{14}$

Total duration of treatment is reported by several authors to be of clinical significance in patients treated by chemoradiation therapy. ${ }^{17-22}$ Scheduling of HDR implant also carried a prognostic significance for local control in patients with locally advanced disease. Whenever patient's anatomy allows brachytherapy should be instituted in third week of EBRT. $^{23}$
In the current study majority of the patients presented with advanced disease, most patients being stage IIB, so brachytherapy was instituted in last week of EBRT schedule allowing for the tumor to shrink and to achieve a good applicator geometry. Only 10 patients were able to complete the entire treatment in less than 50 days, 25 patients were able to complete in 50-60 days, and 15 took more than 60 days to complete treatment. The patients who could not complete treatment in 50 days had delays due to hematologic toxicity related to chemotherapy, leading to interruptions in treatment or missed chemotherapy cycles.

Response of cervical mass and toxicity was evaluated at end of treatment, at 4 and 8 weeks. Though patients receiving doublet chemotherapy regimen had slightly more pronounced vaginal mucosal reactions, there was a trend of improvement of these effects at four week and eight week follow up.

It is well known that cervical cancer is a one of the leading problems for lower socio-economic strata, which are already resource-limited. ${ }^{24,25}$ This treatment strategy provides a cost-effective approach. The strategy can be adapted by radiation facilities designed to offer this curative treatment to large population. It is effective in this center as the patients are coming from far flung areas and mostly have to pay on their own. ${ }^{6}$

\section{CONCLUSION}

This series suggests that three fractions of HDR brachytherapy applications are well tolerated by these patients and toxicity is manageable. Although ABS guidelines has suggested to use 4-6 fractions to keep toxicity less, it is being proposed that increasing the fractions beyond 3 may prolong the treatment duration leading to compromised outcome. Also using three fractions is costeffective in resource-limited setting, because patients come from remote areas for treatment and have to pay on their own. Long term disease control and toxicity remains to be seen in this series of patients and will be reported after a longer follow-up.

\section{ACKNOWLEDGEMENT:}

The authors are grateful to Professor Mohamed Manji, Consultant Radiation Oncologist, BC Cancer Agency, Vancouver, Canada for helping them on this protocol.

\section{ETHICAL APPROVAL:}

Approval for this study was take from the Ethical Review Committee of The Aga Khan University.

\section{PATIENTS' CONSENT:}

Informed consent was taken from all the patients treated on this protocol as standard management strategy according to institutional practice. 


\section{CONFLICT OF INTEREST:}

The authors do not have any financial or other conflict of interest to show.

\section{AUTHOR CONTRIBUTION:}

NA: Contributed to the conception and design of the work, drafted the work or revised it critically for important intellectual content.

BMQ: Drafted the work, revised it critically for important intellectual content; gave final approval of the version to be published.

ANA: Gave final approval of the version to be published; drafted the work or revised it critically for important intellectual content.

$\mathrm{AH}$ : Manuscript writing and literature search.

BMK: Reviewed the manuscript to be published, verified the references.

AQJ: Contributed to the conception and design of the work.

\section{REFERENCES}

1. Torre LA, Bray F, Siegel RL, Ferlay J, Lortet-Tieulent J, Jemal A. Global cancer statistics 2012. CA: Cancer J clinicians 2015; 65(2):87-108. doi: 10.3322/caac.21262.

2. GLOBOCAN, Pakistan cancer fact sheet France: International agency for research on cancer; 2018 [Available from: http://gco.iarc.fr/today/data/factsheets/ populations/586-pakistan-fact-sheets.pdf.

3. Koh WJ, Abu-Rustum NR, Bean S, Bradley K, Campos SM, Cho KR, et al. Cervical cancer, version 3.2019, nccn clinical practice guidelines in oncology. I Natl Compr Canc Netw 2019; 17(1):64-84. doi: 10.6004/jnccn.2019.0001.

4. Viswanathan AN, Thomadsen B. American brachytherapy society consensus guidelines for locally advanced carcinoma of the cervix. part I: General principles. Brachytherapy 2012; 11(1):33-46. doi: 10.1016/ j.brachy. 2011.07.003

5. Song S, Rudra S, Hasselle MD, Dorn PL, Mell LK, Mundt AJ, et al. The effect of treatment time in locally advanced cervical cancer in the era of concurrent chemoradio-therapy. Cancer 2013; 119(2):325-31. doi: 10.1002/ cncr.27652.

6. Chuang LT, Temin S, Camacho R, Dueñas-Gonzalez A, Feldman S, Gultekin M, et al. Management and care of women with invasive cervical cancer: American society of clinical oncology resource-stratified clinical practice guideline. J Global Oncolo 2016; 2(5):311-40. doi: 10.1200/JG0.2016.003954. doi: 10.1200/JG0.2016.003954.

7. Health UDo, Services H. Common terminology criteria for adverse events. CTCAE 4.03 2010;

8. Torre LA, Islami F, Siegel RL, Ward EM, Jemal A. Global cancer in women: Burden and trends. Cancer Epidemiol Biomarkers Prev 2017; 26(4):444-57. doi: 10.1158/ 1055-9965. EPI-16-0858.

9. Aziz N, Yousfani S. Pattern of presentation of cervical carcinoma at nuclear institute of medicine and radiotherapy Pakistan. Pakistan J Med Sci 2013; 29(3): 814-7.

10. Hashmi H, Maqbool A, Ahmed S, Ahmed A, Sheikh K, Ahmed A. Concurrent cisplatin-based chemoradiation in squamous cell carcinoma of cervix. Journal of the college of physicians and surgeons-pakistan : JCPSP 2016; 26(4):302-5.

11. Patankar SS, Tergas AI, Deutsch I, Burke WM, Hou JY, Ananth $\mathrm{CV}$, et al. High versus low-dose rate brachytherapy for cervical cancer. Gynecologic Oncology 2015; 136(3):534-41. doi: 10.1016/j.ygyno.2014.12.038. doi: 10.1016/j.ygyno.2014.12.038.

12. Dueñas-González A, Zarbá JJ, Patel F, Alcedo JC, Beslija S, Casanova L, et al. Phase III open-label, randomized study comparing concurrent gemcitabine plus cisplatin and radiation followed by adjuvant gemcitabine and cisplatin versus concurrent cisplatin and radiation in patients with stage IIB to IVA carcinoma of the cervix. J Clinical Oncology 2011; 29(13):1678-85. doi: 10.1200/JCO.2009.25.9663.

13. Souhami L, Corns R, Duclos M, Portelance L, Bahoric B, Stanimir $\mathrm{G}$. Long-term results of high-dose rate brachytherapy in cervix cancer using a small number of fractions. Gynecologic Oncology 2005; 97(2):508-13. doi: 10.1016/j.ygyno.2005.02.015

14. Petereit DG, Pearcey R. Literature analysis of high dose rate brachytherapy fractionation schedules in the treatment of cervical cancer: Is there an optimal fractionation schedule. Int J Radiation Oncolo Biolo Phys 1999; 43(2):359-66. doi: 10.1016/s0360-3016(98)00387-3.

15. Ghosh S, Rao PB, Kotne S. High dose rate brachytherapy in two 9 gy fractions in the treatment of locally advanced cervical cancer-a south indian institutional experience. Asian Pacific J Cancer Prevention 2015; 16(16):7167-70. doi: 10.7314/apjcp.2015.16.16.7167.

16. Viswanathan AN, Beriwal S, De Los Santos JF, Demanes DJ, Gaffney D, Hansen J, et al. American brachytherapy society consensus guidelines for locally advanced carcinoma of the cervix. Part II: high-dose-rate brachytherapy. Brachytherapy 2012; 11(1):47-52. doi: 10.1016/j. brachy.2011.07.002.

17. Shaverdian N, Gondi V, Sklenar KL, Dunn EF, Petereit DG, Straub MR, et al. Effects of treatment duration during concomitant chemoradiation therapy for cervical cancer. Int J Radiation Oncolo Biolo Phys 2013; 86(3):562-8. doi: 10.1016/j.jjrobp.2013.01.037.

18. Perez CA, Grigsby PW, Castro-Vita H, Lockett MA. Carcinoma of the uterine cervix I. Impact of prolongation of overall treatment time and timing of brachytherapy on outcome of radiation therapy. Int J Radiation Oncolo Biolo Phys1995; 32(5):1275-88. doi: 10.1016/0360-3016 (95)00220-S.

19. Fyles A, Keane TJ, Barton M, Simm J. The effect of treatment duration in the local control of cervix cancer. Radiother Oncolo1992; 25(4):273-9. doi: 10.1016/0167- 8140(92) 90247-r.

20. Chen SW, Liang JA, Yang SN, Ko HL, Lin FJ. The adverse effect of treatment prolongation in cervical cancer by high-dose-rate intracavitary brachytherapy. Radiother Oncol 2003; 67(1):69-76. doi: 10.1016/s0167-8140(02)00439-5.

21. Nugent EK, Case AS, Hoff JT, Zighelboim I, DeWitt LL, Trinkhaus $\mathrm{K}$, et al. Chemoradiation in locally advanced cervical carcinoma: an analysis of cisplatin dosing and other clinical prognostic factors. Gynecologic Oncolo 2010; 116(3):438-41. doi: 10.1016/j.ygyno.2009.09.045.

22. Jemu $M$, van Wijk L, Parker M, Jones G. Tumour and treatment factors influencing the outcome of chemo-radiation in 
stage IIB cervical cancer: A single institution experience. Southern African J Gynaecolo Oncolo 2018; 10(1):5-10. doi.org/10.1080/20742835.2018.1441694.

23. Nag S, Erickson B, Thomadsen B, Orton C, Demanes JD, Petereit $\mathrm{D}$. The American brachytherapy society recommendations for high-dose-rate brachytherapy for carcinoma of the cervix. Int J Radiat Oncol Biol Phys 2000; 48(1):201-11. doi: 10.1016/s0360-3016(00)00497-1.

24. Bhurgri Y. Karachi cancer registry data--implications for the national cancer control program of pakistan. Asian Pac J Cancer Prev 2004; 5(1):77-82.

25. Jemal A, Center MM, DeSantis C, Ward EM. Global patterns of cancer incidence and mortality rates and trends. Cancer Epidemiology and Prevention Biomarkers 2010; 19(8): 1893-907. doi: 10.1158/1055-9965.EPI-10-0437. 